\title{
Die Rolle neuer „pro-resolving“ Lipidmediatoren bei entzündlichen Lungenerkrankungen
}

\author{
Pro-Resolving Lipid Mediators in Inflammatory Lung Diseases
}

Autoren

Institute
O. Eickmeier ${ }^{1}$, J. N. Hilberath ${ }^{2}$, S. Zielen ${ }^{1}$, O. Haworth ${ }^{3}$

Die Institutsangaben sind am Ende des Beitrags gelistet. eingereicht 20.9. 2010

akzeptiert nach Revision 14. 10.2010

\section{Bibliografie}

DOI http://dx.doi.org/

$10.1055 / \mathrm{s}-0030-1255913$

Online-Publikation: 29.11.2010

Pneumologie 2011; 65:

149-158 @ Georg Thieme

Verlag KG Stuttgart · New York

ISSN 0934-8387

\section{Korrespondenzadresse}

Dr. med. Olaf Eickmeier

Zentrum für Kinder- und

Jugendmedizin

Allergologie, Pneumologie und

Mukoviszidose

Klinikum der Johann Wolfgang

Goethe-Universität

Theodor-Stern-Kai 7

60590 Frankfurt/Main

olaf.eickmeier@kgu.de

\section{Zusammenfassung \\ $\nabla$}

Erkrankungen mit unkontrollierter pulmonaler Entzündung stellen eine wesentliche medizinische Herausforderung, aber auch ökonomische Belastung für unsere Gesellschaft dar. Derzeit existieren keine Therapeutika, die diese Art der pathologischen Entzündungen gezielt kontrollieren. Kürzlich wurde eine neue Gruppe von antientzündlichen und proresolutionären Lipidmediatoren entdeckt: Lipoxine, Resolvine, Protektine und Maresine. Diese Lipidmediatoren werden enzymatisch aus den mehrfach ungesättigten Fettsäuren (PUFAs) Arachidonsäure (AA), Docosahexaensäure (DHA) und Eicosapentaensäure (EPA) gebildet, welche seit langem bekannte positive Wirkungen auf diverse Krankheitsverläufe haben können. Diese neuen Lipidmediatoren zeigen in vitro und in vivo in murinen pulmonalen Entzündungsmodellen vielversprechende antiinflammatorische Wirkungen und könnten eine entscheidende Rolle in der Therapie von vielen entzündlichen Lungenerkrankungen, wie z.B. des Asthmas bronchiale, der cystischen Fibrose (CF) und des akuten Lungenversagens spielen.

\section{Einleitung}

$\nabla$

Endogene und molekulare Mechanismen, die eine akute Entzündungsreaktion kontrollieren, sind Gegenstand aktueller Forschung. Unkontrollierte Entzündung spielt eine wichtige Rolle in der Pathogenese vieler Erkrankungen. Die Identifikation endogener Kontrollmechanismen als Reaktion auf einen entzündlichen Stimulus ist hierbei von entscheidender Bedeutung [1].

Die Rückbildung oder Resolution einer Entzündung ist ein aktiver Prozess, der durch eine Vielzahl molekularer und zellulärer Mechanismen gesteuert wird. In den meisten Fällen bildet sich eine akute Entzündung spontan zurück und es

\section{Abstract \\ $\nabla$}

Uncontrolled inflammation of the lung contributes to the major medical and economic burden on healthcare, and the need for therapeutics to dampen pathological inflammation is largely unmet. Recently, a new genus of anti-inflammatory/ pro-resolving lipid mediators has been identified: Lipoxins, resolvins, protectins and maresins. These compounds are enzymatically derived from the polyunsaturated fatty acids (PUFAs) arachidonic acid (AA), docosahexaenoic acid (DHA), and eicosapentaenoic acid (EPA) that have long been known to have beneficial health properties. These mediators have potent anti-inflammatory effects in vitro and in vivo in murine models of lung inflammation. Therefore, this group of compounds carries considerable therapeutic potential for the treatment of many inflammatory lung diseases including asthma, cystic fibrosis and acute lung injury.

wird im Gewebe der Zustand der Homöostase wiederhergestellt. Resolution der Entzündung ist jedoch nicht nur die passive Auflösung von proinflammatorischen Signalen und Mediatoren am Ort des Entzündungsgeschehens, sondern vielmehr eine straff organisierte und vielschichtige, aktive Antwort des Körpers [2]. Diese Antwort hat ihre Ursprünge schon zum Zeitpunkt der akuten Inflammation. Bereits in den ersten Minuten bis Stunden kommt es in akut verletzten, infizierten oder entzündeten Geweben zur Biosynthese chemischer Mediatoren, die neben antientzündlichen Eigenschaften die Resolution der Entzündung fördern [3]. Diese gegenregulatorischen Signale kontrollieren das Ausmaß der entzündlichen 
Tab. 1 Zelluläre Wirkmechanisnem der Lipoxine, Resolvine und Protektine.

\begin{tabular}{|c|c|c|c|c|}
\hline Mediator & Zielzelle & Wirkung & Spezie & Referenz \\
\hline \multirow[t]{13}{*}{ Lipoxin $\mathrm{A}_{4}$} & PMN & hemmt PMN-Chemotaxis & Mensch & [17] \\
\hline & PMN & hemmt transendotheliale Migration & Mensch & {$[85,86]$} \\
\hline & PMN & hemmt transepitheliale Migration & Mensch & [85] \\
\hline & PMN & hemmt ROS-Synthese & Mensch & [87] \\
\hline & PMN & hemmt azurophile Degranulation & Mensch & [88] \\
\hline & Eos & hemmt eosinophile Migration & Mensch & [89] \\
\hline & T-Zellen & hemmt TNF-Ausschüttung & Mensch & [90] \\
\hline & NK-Zellen & hemmt Zytotoxizität der NK-Zellen & Mensch & [91] \\
\hline & Makrophagen & steigert Aufnahme apoptotischer PMNs & Mensch & [92] \\
\hline & Dendritische Zellen & hemmt IL-12-Produktion & Maus & [93] \\
\hline & Enterozyten & hemmt TNF induzierte IL-8-Ausschüttung & Maus & [94] \\
\hline & Endothelzellen & stimuliert die Prostacyclinausschüttung & Mensch & [95] \\
\hline & Epithelzellen & hemmt Zytokinausschüttung & Mensch & [96] \\
\hline \multirow[t]{8}{*}{ Resolvin E1 } & PMN & hemmt transendotheliale Migration & Mensch & [97] \\
\hline & Makrophagen & steigert Aufnahme apoptotischer PMNs & Maus & [98] \\
\hline & \multirow[t]{5}{*}{ Dendritische Zellen } & hemmt Migration & Maus & [25] \\
\hline & & hemmt IL-12-Ausschüttung & Maus & [25] \\
\hline & & hemmt IL-23-Ausschüttung & Maus & [99] \\
\hline & & hemmt IL-6-Ausschüttung & Maus & [99] \\
\hline & & hemmt TNF-Ausschüttung & Maus & [99] \\
\hline & T-Zellen & steigert CCR5-Expression & Mensch & [100] \\
\hline Resolvin D1 & Mikroglia & hemmt IL-1 $\beta$-Auschüttung & Maus & [31] \\
\hline \multirow[t]{2}{*}{ Resolvin D2 } & PMN & hemmt PAF induziertes CD62L-Shedding & Maus & [33] \\
\hline & & hemmt Adhäsion am Gefäßendothel & Maus & [33] \\
\hline \multirow[t]{4}{*}{ Protektin D1 } & T-Zellen & steigert CCR5-Expression & Mensch & [100] \\
\hline & & hemmt TNF- $\alpha$ - und IFN- $\gamma$-Ausschüttung & Mensch & [101] \\
\hline & PMN & hemmt PMN-Transmigration & Mensch & [102] \\
\hline & Mikroglia & hemmt IL-1 $\beta$-Ausschüttung & Maus & {$[36,103]$} \\
\hline
\end{tabular}

Reaktion und führen letztendlich zum Prozess der Katabasis, dem aktiv gesteuerten Abklingen der Entzündung.

Störungen in der Synthese dieser protektiven Substanzen können den Schweregrad von akuten und chronischen entzündlichen Erkrankungen verstärken [4-8]. Diese Übersichtsarbeit informiert über die von den $\omega-6$ und $\omega-3$ mehrfach ungesättigten Fettsäuren (PUFAs) abgeleiteten Lipidmediatoren und deren Rolle bei der Steuerung der Resolution pulmonaler Entzündung.

\section{Lipidmediatoren der w-6 PUFA Arachidonsäure $\nabla$}

\section{Lipoxine}

Lipoxine (LXs) waren die ersten Lipidmediatoren mit antientzündlicher und proresolutionärer Wirkung, die beschrieben werden konnten. LXs sind Produkte des Arachidonsäurestoffwechsels (C20:4) [9]. Sie sind strukturell und funktionell von den Prostaglandinen (PGs), Thromboxanen (TXs) und Leukotrienen (LTs) zu unterscheiden. Indem Lipoxine neutrophile und eosinophile transendotheliale und transepitheliale Migration hemmen, wirken sie antiinflammatorisch und unterstützen die Resolution der Entzündung ( $\bullet$ Tab. 1).

Darüber hinaus hemmen sie die Bildung sog. reaktiver Sauerstoffverbindungen und Radikale (ROS) [10] ( $\bullet$ Abb. 1).

LXs werden am Ort der Entzündung durch einen bidirektionalen und transzellulären Prozess synthetisiert, indem ihre Zwischenprodukte von Zelle zu Zelle weitergereicht werden [11] (๑ Abb. 2).

Ein Schlüsselenzym für die Produktion von LXs ist die 15-Lipoxygenase (15-LO), die u.a. in den Schleimhäuten der Atemwege lokalisiert ist und von vielen Zelltypen wie Epithelzellen,
Makrophagen und Eosinophilen in der entzündeten Lunge gebildet wird. 15-LO verwandelt Arachidonsäure zu einem instabilen Intermediat, genannt 15(S)-HydroperoxyeicosatetraenSäure (15(S)-H(p)ETE), welches durch 5-LO zu LX umgewandelt wird. In der Lunge erfolgt die LX-Biosynthese, wenn eindringende Neutrophile (PMNs) oder Eosinophile (Eos) mit Epithelzellen der entzündeten Atemwege interagieren ( $\bullet$ Abb.2). Lipoxine können aber auch in Blutgefäßen durch Zell-Zell-Interaktionen zwischen Leukozyten über die 5-LO und Thrombozyten über die 12-LO generiert werden. Die humane Thrombozyten 12-LO fungiert dabei als LX Synthase, die aus Leukotrien $\mathrm{A}_{4}\left(\mathrm{LTA}_{4}\right)$ LX bildet ( $\bullet$ Abb. 2) [12].

\section{Aspirin-getriggerte Lipoxine}

Epimere von Lipoxin werden im Verlauf einer Entzündungsreaktion in Anwesenheit von Acetylsalicylsäure (ASA [Aspirin ${ }^{\circledR}$ ]) gebildet [13]. ASA hemmt durch Acetylierung die Cyclooxygenase 2 (COX-2) und damit die Prostaglandinsynthese. Allerdings bleibt das Enzym aktiv und kann weiterhin Arachidonsäure zu 15(R)Hydroxyeicosatetraensäure (15(R)-HETE) umwandeln. 15(R)HETE kann dann als Substrat für leukozytäre 5-LO fungieren und zu 15-epimer-Lipoxin umgewandelt werden ( $\bullet$ Abb.2). 15(R)HETE kann auch über den Cytochrom P450 Stoffwechselweg in Epithelzellen aus der Arachidonsäure synthetisiert werden [14] ( Abb. 2). Eine kürzlich veröffentlichte Studie konnte zeigen, dass Statine (Lovastatin) die Synthese von 15-epi-LXs fördern und dadurch den Heilungsverlauf von Säure-induzierter akuter Lungenverletzung positiv beeinflussen [15]. 


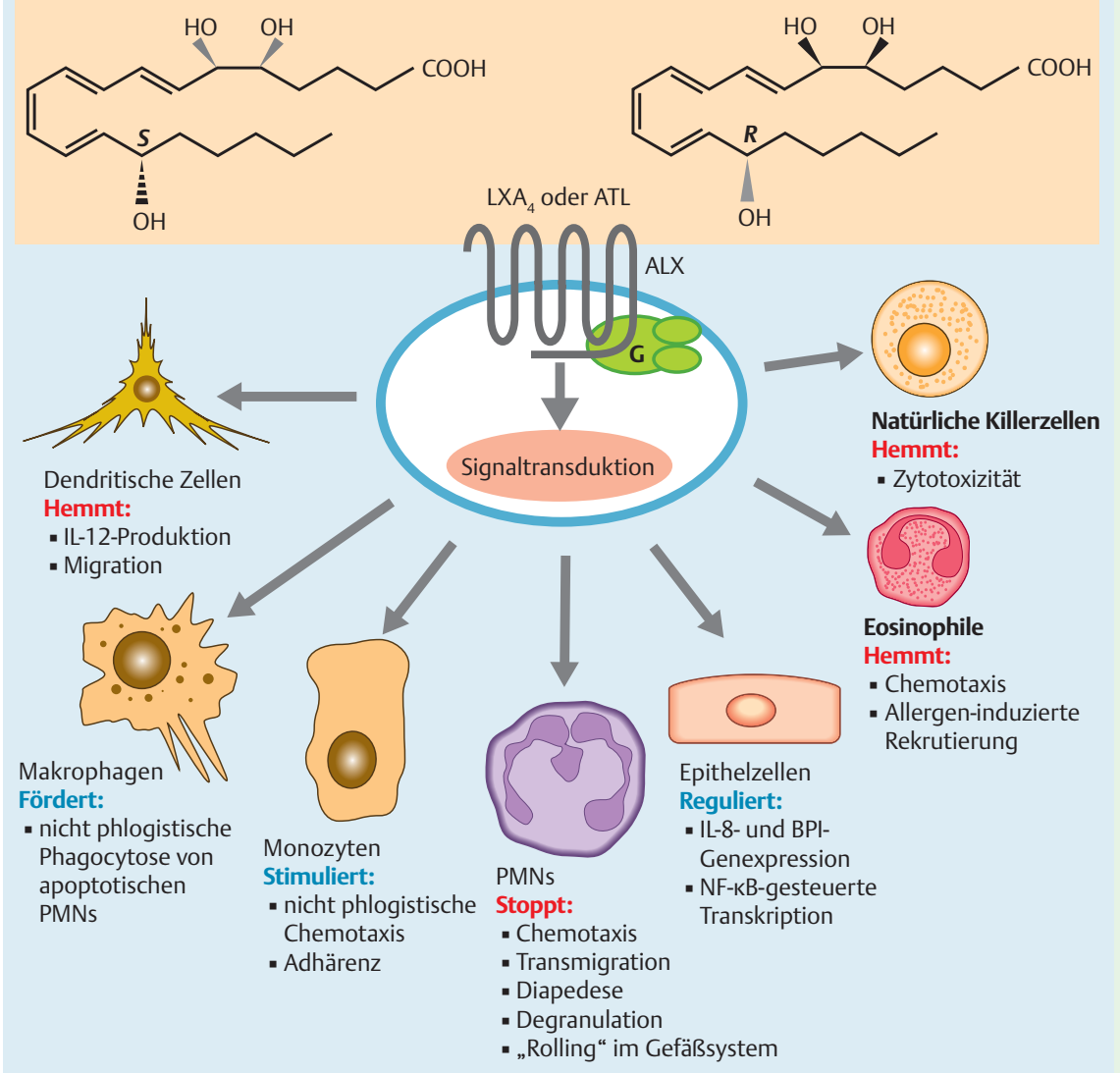

Abb. 1 Spezifische Stoffwechselwege und Wirkungen der Lipoxine. Lipoxin $\mathrm{A}_{4}\left(\mathrm{LXA}_{4}\right)$ und Aspiringetriggertes Lipoxin $A_{4}(A T L)$ interagieren mit dem spezifischen Zelloberflächenrezeptor ALX. Die Aktivierung dieses Rezeptors durch $L X A_{4}$ und ATL verursacht zellspezifische Effekte, wie Inhibition der Migration dendritischer Zellen, Hemmung von IL-12-Ausschüttung, Stimulation von Makrophagen zur Aufnahme apoptotischer PMNs, Förderung der Monozytenmigration, Inhibition der Neutrophileneinwanderung und Degranulation, Reduktion der IL-8-Ausschüttung und BPI (bactericidal/permeability increasing Protein) von Epithelzellen, Inhibition eosinophiler Chemotaxis und Inhibition NK-Zellenvermittelter Zytotoxizität.

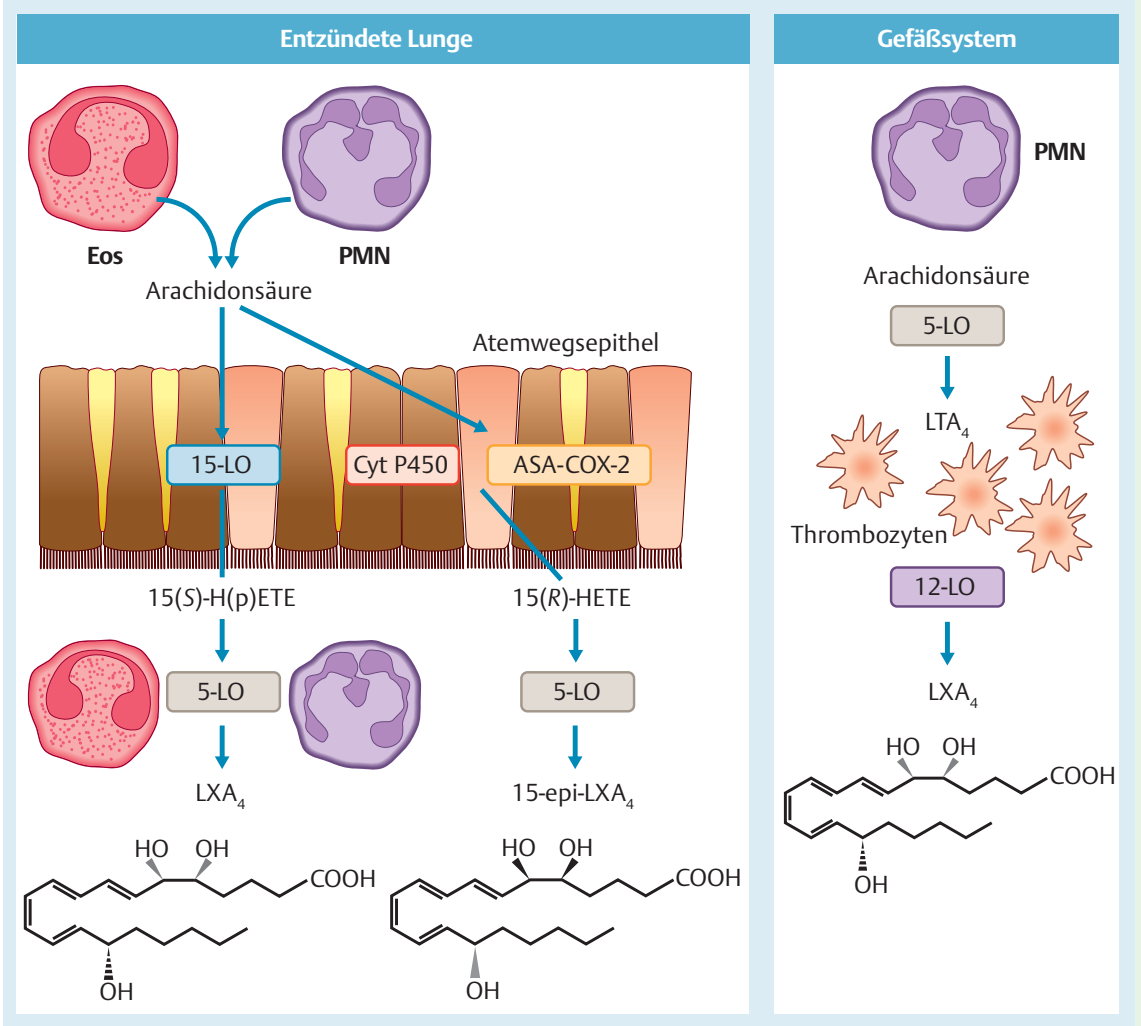

Abb. 2 Bildung der Lipoxine. Lipoxin $\mathrm{A}_{4}\left(\mathrm{LXA}_{4}\right)$ kann in der entzündeten Lunge oder auch in Blutgefäßen gebildet werden. In der Lunge wandelt die epitheliale 15-Lipoxygenase (15-LO) Arachidonsäure zu 15(S)-H(p)ETE um, welches durch leukozytäre 5-Lipoxygenase (5-LO) zu LXA 4 transformiert wird. Die 15-epi-LX-Synthese wird durch 5-LO vermittelte Umwandlung von 15(R)-HETE durch Cytochrom p450 oder durch die Aspirin ${ }^{\circledR}$ (ASA)-acetylierte COX-2 katalysiert. Im Gefäßsystem können LXs durch Zusammenwirken von aktivierten Leukozyten (5-LO) und Thrombozyten (12-LO) gebildet werden. 


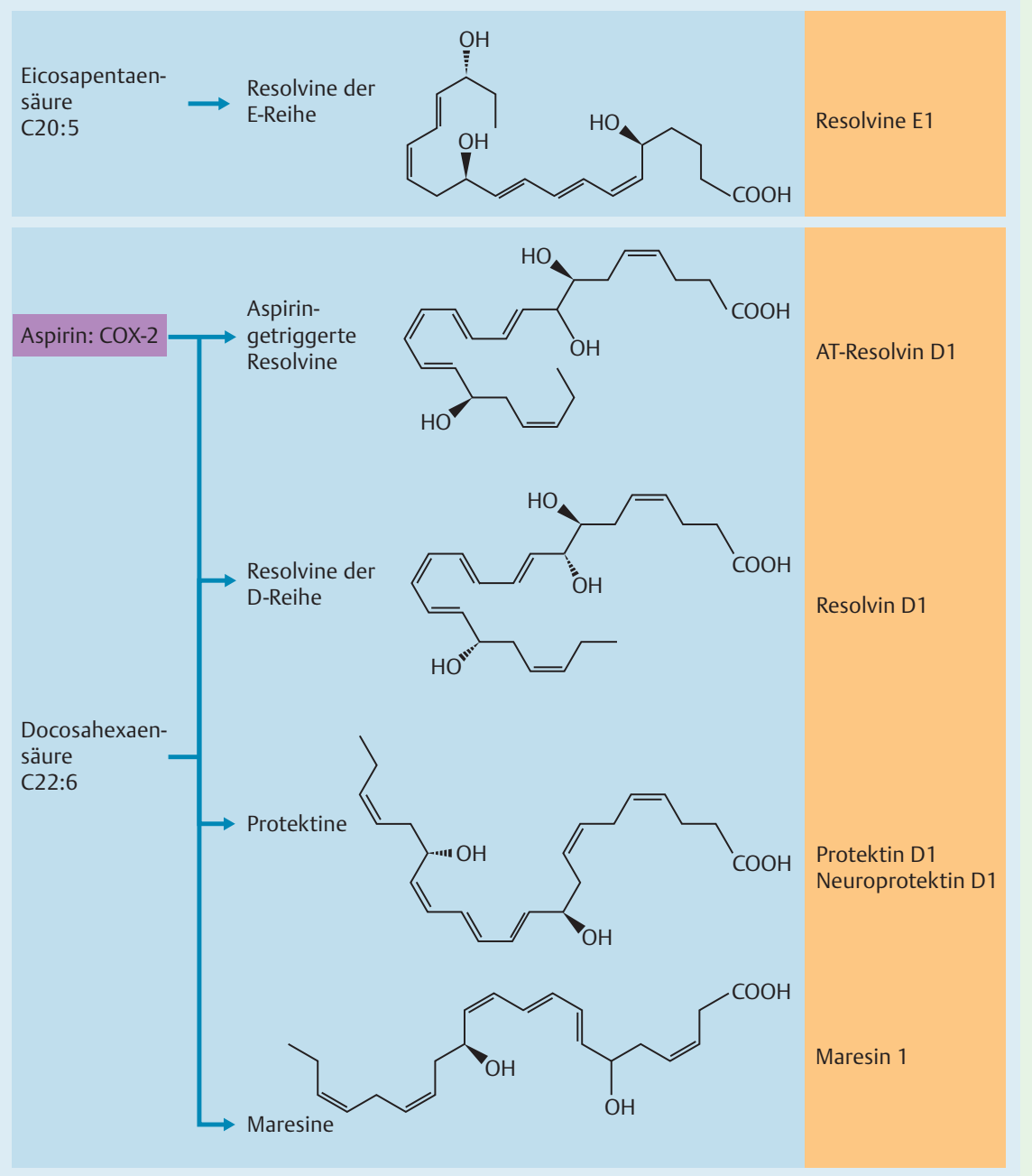

Abb. 3 Bildung der Resolvine, Protektine und Maresine. Resolvine, Protektine und Maresine sind durch Zell-Zell-Interaktionen enzymatisch aus $\omega-3$ PUFAs gebildete aktive Lipidmediatoren. Die Resolvine der E-Reihe werden aus Eicosapentaensäure (EPA) gebildet, aus denen z. B. RvE1 hervorgeht. Die Resolvine der D-Reihe werden aus Docosahexaensäure (DHA) gebildet. Ferner kann aus DHA in Anwesenheit von durch Aspirin ${ }^{\circledR}$ acetylierter COX-2 die Gruppe der Aspirin-getriggerten Resolvine z. B. AT-RvD1 gebildet werden. DHA kann auch als Vorläufer zur Bildung von Protektinen und Maresinen dienen.

Ähnlich anderen Autacoiden werden Lipoxine rasch gebildet und ebenso schnell inaktiviert. Das Enzym 15-Hydroxyprostaglandin-Dehydrogenase (15-PGDH) metabolisiert Lipoxine zu 15oxo- $\mathrm{LXA}_{4}$ oder 5-oxo- $\mathrm{LXB}_{4}[16]$. Diese inaktivierten Lipoxine besitzen keine gegenregulatorischen Effekte mehr. Der LipoxinMetabolismus ist stereospezifisch, sodass 15-epi-Lipoxine wesentlich weniger effizient metabolisiert werden. Das erhöht ihre biologische Halbwertszeit und damit auch ihre Wirkdauer [17]. Somit sind die pharmakologischen Eigenschaften von ASA zum einen auf die Fähigkeit zurückzuführen, die proinflammatorische Wirkung der Prostaglandine zu hemmen, zum anderen wird die Bildung neuer antiinflammatorischer Lipidmediatoren wie z.B. 15-epi-LXs gefördert. Die ASA-getriggerte Bildung von gegenregulatorischen Lipidmediatoren aus der Arachidonsäure ist auch für die aktiven Lipidmediatoren der $\omega$-3 PUFAs Eicosapentaensäure (C20:5) und Docosahexaensäure (C22:6) beschrieben.

\section{Lipidmediatoren der $\omega$-3 PUFAs DHA und EPA \\ $\nabla$}

\section{Resolvine}

Die Vorteile der Einnahme von $\omega-3$ PUFAs sind heute so gut untersucht, dass sie einen wesentlichen Bestandteil einer gesunden Diät darstellen [18]. Omega-3-Fettsäuren sind in Fischölen konzentriert und eine fischölreiche Diät verringert die Prävalenz oder Krankheitsschwere von Asthma bronchiale, cystischer Fibrose (CF), Herzerkrankungen und Krebs [19-21].
Resolvine sind Interaktionsprodukte der Resolutionsphase von Entzündungen. Sie wurden erstmals in sich spontan auflösenden entzündlichen Exsudaten identifiziert. Resolvine sind stereospezifische kleine Moleküle, die sich enzymatisch von den $\omega$-3-Fettsäuren EPA und DHA ableiten [22,23] $\bullet$ Abb. 3).

Ähnlich den Lipoxinen können Resolvine aus 5-LO-katalysierten Reaktionen mit biosynthetischen Zwischenprodukten, die zwischen verschiedenen Zellen ausgetauscht werden, entstehen. Abhängig von der jeweiligen $\omega$-3 Fettsäure können Resolvine in eine D-Reihe (DHA, C22:6) und eine E-Reihe (EPA, C20:5) eingeteilt werden ( Abb. 3, $\bullet$ Tab. 1). Die Bildung dieser Substanzen wird durch die Nahrungszusammensetzung beeinflusst.

\section{Resolvine der E-Reihe}

Resolvin E1 (RvE1) wird enzymatisch von der $\omega$-3 Fettsäure EPA abgeleitet und wirkt sowohl in vitro, als auch in vivo stark entzündungshemmend und proresolutionär ( $\bullet$ Abb. 3, $\bullet$ Tab. 1,2) $[22,23]$.

RvE1 dient als Ligand für den G-Protein-gekoppelten Rezeptor (GPCR) ChemR23, der auf myeloiden dendritischen, aber auch Mukosaepithelzellen präsentiert wird. Ursprünglich wurde ChemR23 als Rezeptor für das Peptid Chemerin identifiziert [24], doch auch RvE1 ist ein Agonist für ChemR23, der zellartspezifische gegenregulatorische Signale übermittelt. So verringert die Interaktion von RvE1-ChemR23 durch Phosphorylierung von Akt und Protein S6 eine durch proinflammatorische Signale gesteuerte NF-KB-Aktivierung $[25,26]$. Weiterhin wirkt RvE1 anta- 
Tab. 2 Lipoxine, Resolvine und Protektine und ihre Wirkung auf die Inflammation.

\begin{tabular}{|c|c|c|c|c|}
\hline Mediator & Erkrankung & Spezie & Wirkung & Referenz \\
\hline \multirow[t]{10}{*}{ Lipoxin $\mathrm{A}_{4}$} & Asthma & Maus & $\begin{array}{l}\text { hemmt eosinophile Anziehung } \\
\text { dämpft bronchiale Hyperreagibilität }\end{array}$ & {$[51]$} \\
\hline & Peritonitis & Maus & $\begin{array}{l}\text { hemmt PMN-Migration } \\
\text { erhöht Makrophagenclearance }\end{array}$ & {$[98,102]$} \\
\hline & Colitis & Maus & $\begin{array}{l}\text { verbessert Gewichtsentwicklung } \\
\text { verringert Colitis }\end{array}$ & [104] \\
\hline & Ischämie/Reperfusion & Maus & hemmt sekundäre Lungenschädigung & [105] \\
\hline & Angiogenese & Maus & hemmt vaskuläre Neogenese & [106] \\
\hline & Hyperalgesie & Ratte & hemmt Schmerzen & [107] \\
\hline & Cystische Fibrose & Maus & $\begin{array}{l}\text { reduziert PMN-Infiltration } \\
\text { reduziert Bakterienlast }\end{array}$ & [4] \\
\hline & Periodontitis & Kaninchen & $\begin{array}{l}\text { hemmt PMN-Migration } \\
\text { verhindert Gewebs-und Knochenverlust }\end{array}$ & [108] \\
\hline & Korneale Wundheilung & Maus & fördert die korneale Wundheilung & [109] \\
\hline & $\begin{array}{l}\text { Bleomycin-induzierte } \\
\text { Lungenfibrose }\end{array}$ & Maus & $\begin{array}{l}\text { verringert Fibroblastenproliferation und Matrix- } \\
\text { ablagerungen }\end{array}$ & [63] \\
\hline \multirow[t]{6}{*}{ Resolvin E1 } & Peritonitis & Maus & $\begin{array}{l}\text { hemmt PMN-Migration } \\
\text { hemmt die Zytokinfreisetzung } \\
\text { fördert die Abwanderung von Makrophagen über } \\
\text { das Lymphsystem }\end{array}$ & {$[110,111]$} \\
\hline & Retinopathie & Maus & hemmt vaskuläre Neogenese & [112] \\
\hline & Colitis & Maus & $\begin{array}{l}\text { reduziert Dünndarmentzündung } \\
\text { reduziert Zytokinfreisetzung } \\
\text { erhöht Überlebensrate }\end{array}$ & {$[113,114]$} \\
\hline & Periodontitis & Kaninchen & $\begin{array}{l}\text { beugt Knochen- und Bindegewebsverlust vor } \\
\text { regeneriert Knochen- und Bindegewebe }\end{array}$ & {$[110,111]$} \\
\hline & Asthma & Maus & hemmt eosinophile Infiltration & [49] \\
\hline & Akute Lungenschädigung & Maus & $\begin{array}{l}\text { fördert Resolution, reduziert IL-6, }-17,-23 \text { und erhöht } \\
\text { LXA }_{4} \text { - und IFN-Y-Spiegel } \\
\text { erniedrigt Leukozyteninfiltration und schützt gegen } \\
\text { Pneumonie }\end{array}$ & [82] \\
\hline \multirow[t]{4}{*}{ Protektin D1 } & Retinopathie & Maus & hemmt vaskuläre Neogenese & [112] \\
\hline & Asthma & Maus & $\begin{array}{l}\text { hemmt eosinophile Infiltration } \\
\text { reduziert bronchiale Hyperreagibilität }\end{array}$ & [84] \\
\hline & Peritonitis & Maus & $\begin{array}{l}\text { reduziert PMN-Infiltration } \\
\text { reguliert Zytokinproduktion } \\
\text { fördert Makrophagenabwanderung über das Lymph- } \\
\text { system }\end{array}$ & {$[98,102]$} \\
\hline & $\begin{array}{l}\text { Niere } \\
\text { Ischämie/Reperfusion }\end{array}$ & Maus & reduziert renalen Ischämie-/Reperfusionsschaden & {$[30]$} \\
\hline Resolvin D1 & $\begin{array}{l}\text { Niere } \\
\text { Ischämie/Reperfusion }\end{array}$ & Maus & reduziert Nierenschaden durch Ischämie/Reperfusion & $\begin{array}{l}{[30]} \\
{[33]}\end{array}$ \\
\hline Resolvin D2 & $\begin{array}{l}\text { Peritonitis } \\
\text { Mikrobielle Sepsis }\end{array}$ & $\begin{array}{l}\text { Maus } \\
\text { Maus }\end{array}$ & $\begin{array}{l}\text { reduziert PMN-Infiltration } \\
\text { reduziert Freisetzung proinflammatorischer Zytokine }\end{array}$ & [82] \\
\hline
\end{tabular}

gonistisch und partiell agonistisch auf den Leukotrien $\mathrm{B}_{4}\left(\mathrm{LTB}_{4}\right)$ Rezeptor BLT1 [27].

\section{Resolvine der D-Reihe}

DHA ist eine $\omega$-3-Fettsäure, die besonders im Nervengewebe angereichert ist und neuroprotektiv wirkt [28]. DHA kann über die Interaktion von 15-LO und 5-LO zu den 17S konfigurierten Resolvinen der D-Reihe oder, ähnlich den Lipoxinen, über die ASAacetylierte COX-2 und 5-LO zu den 17R konfigurierten Resolvinen der D-Reihe katalysiert werden ( $\bullet$ Abb. 2) [10]. Resolvin D (RvD) hemmt die neutrophile Infiltration und Migration und wirkt protektiv in murinen Ischämie-Reperfusionsmodellen und Modellen bakterieller Peritonitis ( Tab. 1,2) [29-33]. Ähnlich den Resolvinen der E-Reihe und den Lipoxinen können verschiedene Rezeptoren mit den Resolvinen der D-Reihe interagieren. Der GProtein-gekoppelte Rezeptor GPR32 und der Lipoxinrezeptor ALX wurden als Rezeptoren für RvD identifiziert [34].

\section{Protektine}

Protektin (P)D1 ist ein Docosatrien, das analog zu den aus der AA gebildeten Lipoxinen über eine 15-LO-katalysierte Reaktion aus der DHA synthetisiert wird $[28,29]$. Dieses Docosatrien wurde, nachdem seine Bildung durch Gliazellen im DHA-reichen Gewebe des Gehirns entdeckt wurde, Neuroprotectin D1 genannt. PD1 schützt gegen ischämische Hirnschädigung und Morbus Alzheimer $[28,35,36]$. Ferner moduliert PD1 Zellantworten zum Schutz gegen Nieren- und Leberschädigung ( $\checkmark$ Tab. 1,2) [30,37]. PD1 bindet mit hoher Affinität an menschliche neutrophile Zellen $[28,38]$. Der Rezeptor ist noch nicht beschrieben.

\section{Maresine}

Kürzlich wurde eine neue Substanzgruppe entdeckt, die von aktivierten Makrophagen gebildet wird, wenn DHA in neue 7,14-Dihydroxydocosa-4Z,8,10,12,16Z,19Z-hexaensäure-Produkte, sogenannte Maresine (Makrophagen-Mediatoren in sich auflösen- 
der Inflammation), umgewandelt wird. So wie Resolvine und Protektine zeigen auch Maresine (MaRs) starke antiinflammatorische Wirkungen [39]. Makrophagen stellen Schlüsselzellen bei der Auflösung (Resolution) von Entzündung, der Reparatur von Gewebeschäden und der Wiederherstellung der Homöostase dar [40]. Nicht zuletzt, weil Makrophagen eine essenzielle Bedeutung bei der Beseitigung von apoptotischen und nekrotischen Zellen, beim Umschließen von mikrobiellen Partikeln und Hemmung neutrophiler Invasion am Ort der Entzündung haben, könnten Maresine neben einer Verkürzung des Resolutionsintervalles potenziell auch von einer Schädigung bedrohte Gewebe schützen.

\section{Die Rolle der neuen Lipidmediatoren bei entzündlichen Lungenerkrankungen \\ $\nabla$}

\section{Lipoxine und pulmonale Entzündung \\ Asthma}

Asthma ist eine chronische Atemwegsentzündung, die durch Infiltration von verschiedenen Zellen u. a. Eosinophilen und T-Lymphozyten charakterisiert ist. Diese Zellen setzen Zytokine, Chemokine, aber auch proinflammatorische Lipidmediatoren frei, wie z.B. Leukotriene, die maßgeblich an der Krankheitsschwere beteiligt sind. Der Umbau des Atemwegsepithels, der Basalmembran und der glatten Muskulatur, auch Remodeling genannt, führt in einigen Fällen zur fixierten Atemwegsobstruktion $[41,42]$.

Unter den Asthmatikern leiden ca. 5\% an einer schweren Manifestation ihrer Erkrankung, die sich durch anerkannte Therapiestrategien nicht bessert [43]. Eine verminderte Synthese von Lipoxinen ist mit schwerer Atemwegsentzündung assoziiert [44]. So ist für das Aspirin-getriggerte Asthma bronchiale eine schwerere Verlaufsform kennzeichnend, die mit einer verminderten Lipoxinsynthese im Vergleich zum Aspirin-toleranten Asthma einhergeht [45]. Bei schweren Verlaufsformen eines Asthmas wurden signifikant niedrigere Spiegel von $\mathrm{LXA}_{4}$ im Überstand von induziertem Sputum im Vergleich zu milden Verlaufsformen gemessen. Weiterhin konnten niedrige $\mathrm{LXA}_{4}$-Spiegel mit dem Belastungsasthma assoziiert werden $[7,46]$. Im Blut von Asthmatikern mit einer schweren Verlaufsform lässt sich eine deutlich reduzierte Fähigkeit zur Umwandlung von Arachidonsäure zu den durch die 15-LO-katalysierten Produkten, einschließlich 15HETE und $\mathrm{LXA}_{4}$, nachweisen $[5,6]$.

Weiterhin konnte eine erniedrigte $\mathrm{LXA}_{4}$-Bildung bei Patienten mit schwerem Asthma mit erniedrigten mRNA-Spiegeln der Gene, die für die $\mathrm{LXA}_{4}$-Synthese kodieren, korreliert werden [47]. Im Gegensatz hierzu sind 5-HETE, LTB $_{4}$ und die Cysteinyl-Leukotriene (CysLTs) bei schweren Asthmatikern im Blut stark erhöht. Besonders interessant ist die positive Korrelation zwischen Lungenfunktion ( $\mathrm{FEV}_{1} \%$ des Solls) der asthmatischen Studienteilnehmer und $\mathrm{LXA}_{4}$-Spiegeln, die eine Beziehung zur Fähigkeit der Bildung dieser aktiven Lipidmediatoren und Ausprägung der Atemwegsobstruktion beschreibt $[5,6]$.

Die protektiven Eigenschaften der Lipoxine, die Entdeckung ihrer Stoffwechselwege und die strukturelle Beschreibung der 15-epiLipoxine hat die Grundlage für die Entwicklung einer stabilen, der Lipoxinstruktur ähnlichen Substanz geschaffen, die in der Therapie entzündlicher Erkrankungen, wie z.B. des Asthmas bronchiale ihre Anwendung finden könnte. Dass LXs eine entwicklungsfähige therapeutische Strategie in der Behandlung der Atemwegsentzündung darstellen könnten, wurde an Mäusen gezeigt, die ein stabiles $\mathrm{LXA}_{4}$-Analogon erhalten haben, welches $\mathrm{zu}$ reduzierter respiratorischer Inflammation und beschleunigter Entzündungsresolution führte $[48,49]$.

$\mathrm{LXA}_{4}(0,4-2,8 \mathrm{ng} / \mathrm{ml})$ konnte in bronchoalveolärer Lavage (BAL) Flüssigkeit von Patienten mit verschiedenen Lungenerkrankungen, wie Sarkoidose, Pneumonie, Asthma, Alveolitis und Bronchialkarzinom, bestimmt werden [50]. Weiterhin konnte $\mathrm{LXA}_{4}$ in der Pleuraflüssigkeit von Patienten mit systemischer und pulmonaler Inflammation bestimmt werden [48]. Bereits geringe Dosen von $1 \mu \mathrm{g}(\sim 0,05 \mathrm{mg} / \mathrm{kg})$ Lipoxinanalogon und 15-epi-LX, die Ovalbumin (OVA)-sensibilisierten Mäusen vor bronchialer OVA-Provokation verabreicht wurden, verhinderten die Entwicklung allergischer Atemwegsentzündung [51]. Noch vor der Entwicklung von LX-Analoga konnte in einer Untersuchung bei Probanden mit mildem Asthma bronchiale durch die Inhalation von aerosolisiertem $\mathrm{LXA}_{4}$ eine durch Leukotrien C4 ( $\left.\mathrm{LTC}_{4}\right)$ induzierte Bronchokonstriktion geblockt werden. Dies bestätigt die atemwegsprotektive Wirkung von $\mathrm{LXA}_{4}$ bei Asthmatikern. Weiterhin zeigten sich in dieser Untersuchung keine Nebenwirkungen in Bezug auf die normale Atemwegsphysiologie oder den Blutdruck. Bis heute ist diese Pilotstudie die einzige klinische Studie mit LXs [52].

\section{Cystische Fibrose (CF)}

Die cystische Fibrose (CF) ist eine Systemerkrankung, die u.a. durch eine Mutation im „Cystic Fibrosis Transmembrane Conductance Regulator (CFTR)“-Gen begründet ist [53,54]. Bei der CF wird die entzündliche Immunantwort hauptsächlich durch bakterielle Infektionen bestimmt, besonders durch Keime wie Pseudomonas, die zu Gewebsuntergang und schweren Lungenschäden führen [55]. Die Analyse von Biomarkern im induzierten Sputum zeigt eine enorme Erhöhung verschiedener proinflammatorischer Mediatoren mit individuellen Unterschieden [56]. Die inflammatorische Antwort bei CF ist neutrophil dominiert und wird durch neutrophile chemotaktische Mediatoren wie Interleukin 8 (IL-8) und $\mathrm{LTB}_{4}$ gekennzeichnet, die die Freisetzung weiterer proinflammatorischer Mediatoren aus Neutrophilen fördern [57-60].

Unterschiede im Fettsäurestoffwechsel im Vergleich zu Gesunden konnten für CF-Patienten beschrieben werden. Erniedrigte Spiegel von Linolsäure (LA) und DHA wurden in mehreren Untersuchungen bestätigt $[21,61]$. Die Lipoxinspiegel in den Atemwegen einiger CF-Patienten scheinen erniedrigt [4]. Eine aktuelle Untersuchung beschreibt, dass die defekte CFTR-Expression auf Thrombozyten von CF-Patienten zu einer erniedrigten Produktion $(\sim 40 \%)$ von LX im Vergleich zu gesunden Kontrollen führt, wenn diese Thrombozyten mit Neutrophilen inkubiert werden [32].

\section{Lungenfibrose}

Die Fibrosierung der Lunge ist eine gemeinsame Eigenschaft von vielen Lungenerkrankungen. Sie ist durch eine Hyperplasie von Fibroblasten und eine erhöhte Matrixablagerung charakterisiert und ferner mit einer hohen Morbidität und Mortalität vergesellschaftet [62]. Eine kürzlich publizierte Studie konnte in einem Mausmodell bleomycin-induzierter pulmonaler Fibrose zeigen, dass ein als Prophylaxe verabreichtes aspirin-getriggertes Lipoxinanalogon (ATLa) die Entstehung einer Fibrose verhindert. In einem zweiten Szenario bei bereits etablierter Fibrose konnte ATLa als Therapeutikum zusätzlich die Fibroblastenproliferation und Matrixablagerungen reduzieren [63]. 
Akute Lungenverletzung und akutes Lungenversagen

Akute Lungenverletzung (Acute lung injury (ALI)) oder die schwere Form des akuten Lungenversagens (Adult (acute) respiratory distress syndrome (ARDS)) ist ein häufiger Grund für eine intensivmedizinische Versorgung mit der Notwendigkeit der Beatmung. Trotz vieler Fortschritte im Verständnis der Pathophysiologie von ARDS konnten bislang nur wenige Interventionsstrategien den Krankheitsverlauf verbessern [64,65]. Die Aspiration ist eine häufige Ursache für Pneumonien und ALI/ARDS [66]. In den meisten Fällen bildet sich die Pneumonie spontan zurück [67], was die Existenz von endogenen, autoprotektiven Signalwegen nahelegt. Trotz detaillierter Unterteilung der histopathologischen Stadien, die die Rückbildung einer komplett konsolidierten Lungenarchitektur zu einem scheinbar normalen Status beschreiben, gibt es bislang nur wenige Erkenntnisse über die pathophysiologischen Mechanismen, die diesem Prozess der Resolution der pulmonalen Infektion zugrunde liegen. Vor diesem Hintergrund wurde ein Modell der sich spontan auflösenden Entzündung bei akuter pulmonaler Schädigung durch Säure entwickelt, um die endogenen proresolutionären Mechanismen gezielt untersuchen zu können [68]. Die pharmakologische oder gentherapeutische Inhibition der COX-2-Aktivität nach pulmonaler Schädigung reduziert die frühe neutrophile Invasion in die Lunge, führt aber paradoxerweise zu späteren Zeitpunkten zu einer Zunahme der Entzündung. Dies liegt in dem späteren antientzündlichen Effekt der COX-2 begründet, der durch die Bildung von 15-epi-LXA ${ }_{4}$, im Gegensatz zu dem gemeinhin bekannteren proinflammatorischen Effekt, entscheidend zur Genesung nach ALI beiträgt [68]. Das bestätigen Vorarbeiten, die zeigen, dass COX-2 essenziell für die Resolution der Entzündung ist [69].

\section{Resolvine und pulmonale Entzündung}

Epidemiologische Studien bestätigen, dass eine Ernährung, die reich an $\omega$-3-Fettsäuren ist, mit einer niedrigeren Prävalenz von Asthma assoziiert ist [70]. Ferner gibt es Hinweise, dass die Supplementation mit $\omega-3$ PUFAs die bronchiale Entzündung nach spezifischer bronchialer Provokation vermindert [71]. Resolvine wurden in einer Reihe muriner Modelle pulmonaler Entzündung auf ihre entzündungsdämpfende und resolutionsfördernde Wirkung getestet.

\section{Allergische Atemwegsentzündung}

Die Wirkung von RvE1 wurde in einem murinen Modell allergischer Atemwegsentzündung überprüft [49]. Die intravenöse Gabe von RvE1 verminderte die Entstehung allergischer Entzündung und beschleunigte im therapeutischen Szenario die Resolution der Atemwegsentzündung. RvE1 vermittelte seine proresolutionären Effekte, indem es zur Reduktion der Zytokine IL-6, IL17 und IL-23 führte, die mit einem breiten Spektrum unterschiedlicher inflammatorischer Erkrankungen assoziiert sind [72]. IL-17 kommt in asthmatischen Atemwegen vor und kann dort Entzündung hervorrufen und die Mukusproduktion stimulieren [73-75]. Obwohl RvE1 die Resolution förderte, hatte es keinen Effekt auf die $\mathrm{T}_{\mathrm{H}} 2$-Zytokine IL-5 und IL-13, die wohl eher an der Genese der Atemwegsentzündung als an ihrer Aufrechterhaltung beteiligt sind.

Interessanterweise erhöhte die Gabe von RvE1 auch die Spiegel von $\mathrm{LXA}_{4}$. RvE1 und $\mathrm{LXA}_{4}$ besitzen demnach sich überschneidende und ergänzende proresolutionäre Wirkungen, die jedoch durch unterschiedliche Mechanismen erzielt werden [49].
Akute Lungenverletzung und akutes Lungenversagen In vielen Untersuchungen konnten die Vorteile einer fischölhaltigen Ernährung nach ALI/ARDS in Bezug auf Oxygenierung, Beatmungsdauer, Auftreten weiteren Organversagens und Letalität bestätigt werden [76 - 79]. Transgene fat-1 Mäuse können aus $\omega$ 6 -Fettsäuren über die Expression einer $\omega$-3-Fettsäuredesaturase die protektiven $\omega$-3-Fettsäuren bilden und zeigen somit ein erniedrigtes $\omega 6: \omega 3$ PUFAs-Verhältnis in ihren Organen, ohne dass eine diätetische Intervention notwendig ist [80]. Nach Instillation von LPS zeigen fat-1-Mäuse eine deutlich reduziert ausgeprägte pulmonale Schädigung im Vergleich zu Wildtyptieren [81]. Der aus der EPA abgeleitete Lipidmediator RvE1 konnte im Mausmodell eine durch Säure und Bakterien induzierte Lungenschädigung mit Pneumonie abmildern [82]. Trotz der Instillation von Säure und Bakterien (E. coli) in den linken Lungenlappen führte die sich entwickelnde Aspirationspneumonie innerhalb der ersten drei Tage zu einer Letalität von 50\%. Im Gegensatz hierzu überlebten alle Tiere, die vor der pulmonalen Schädigung RvE1 intravenös erhielten. RvE1 konnte die Überlebensrate ebenfalls signifikant verbessern, wenn es erst zwei Stunden nach E. coliApplikation verabreicht wurde. In diesem Modell der Aspirationspneumonie konnte RvE1 die Synthese von proinflammatorischen Mediatoren wie IL-1 $\beta$, IL-6, MIP- $1 \alpha$, MIP-1 $\beta$, KC (entspricht humanem IL-8) und MCP-1 vermindern. Dies könnte von entscheidender Bedeutung sein, da diese Mediatoren in der frühen Phase eines ARDS in erhöhten Konzentrationen in der BALF bei Nichtüberlebenden nachweisbar sind [83]. Obwohl RvE1 keine direkte antimikrobielle Wirkung zeigte, konnte die bakterielle Clearance aus dem Lungengewebe und damit auch die Überlebensrate deutlich verbessert werden. Dies begründet sich möglicherweise in der Regulation der o.g. Zytokine/Chemokine.

\section{Protektine und pulmonale Entzündung \\ Asthma bronchiale}

Protektin D1 (PD1) wird u.a. in den menschlichen Atemwegen gebildet. PD1 und sein biosynthetischer Vorläufer 17S-HDHA konnten im exhalierten Atemkondensat bei Patienten mit Asthma bronchiale, die sich im Rahmen einer Exazerbation in einer Notfallambulanz vorstellten, nachgewiesen werden [84]. In einem Modell der OVA-induzierten allergischen Atemwegsentzündung reduzierte eine im Nanogrammbereich liegende Injektion von PD1 zwei Hauptmerkmale des Asthmas bronchiale: die eosinophile Migration in die Lunge und die bronchiale Hyperreagibilität. Auch wenn PD1 bei bereits fortgeschrittener Entzündung verabreicht wurde, konnte die Resolution der allergischen Atemwegsentzündung beschleunigt werden [84]. Diese Beobachtungen lassen auf eine therapeutische Anwendung für PD1 beim Asthma bronchiale hoffen.

\section{Zusammenfassung}

\section{$\nabla$}

Die Auflösung (Resolution) einer Entzündung ist ein unerlässlicher und kritischer Teil der physiologischen Antwort auf Gewebsverletzung, Infektion und toxische Reize. Resolution ist ein aktiver Prozess und eine Reihe von natürlichen kleinen Molekülen konnte als Mediatoren für die Wiederherstellung der Homöostase im geschädigten Gewebe identifiziert werden. Einblicke in die Biochemie dieser Mediatoren und die Beschreibung ihrer Signalwege ist eine wichtige Möglichkeit zur Entwicklung einer neuen Klasse potenzieller Therapeutika. Außerdem scheinen einige entzündliche respiratorische Erkrankungen, wie z.B. das 
Asthma bronchiale und hier besonders schwere Manifestationen dieser Erkrankung, ursächlich mit einem Defekt in der Bildung dieser neuen Lipidmediatoren verknüpft zu sein. Zusammenfassend kann festgestellt werden, dass stabile Lipoxinanaloga und auf den kürzlich entdeckten Resolvinen und Protektinen basierende Wirkstoffe eine neue vielversprechende Option in der Therapie entzündlicher Lungenerkrankungen darstellen. Es bleibt abzuwarten, ob sich diese neuen Lipidmediatoren im klinischen Alltag bewähren.

\section{Interessenkonflikt}

$\nabla$

Die Autoren geben an, dass kein Interessenkonflikt besteht.

\section{Institute}

Zentrum für Kinder- und Jugendmedizin, Allergologie, Pneumologie und Mukoviszidose, Klinikum der Johann Wolfgang Goethe- Universität Frankfurt a. M.

2 Department of Anesthesiology, Perioperative and Pain Medicine, Brigham and Women's Hospital and Harvard Medical School, Boston, MA, USA

Department of Internal Medicine, Pulmonary and Critical Care Medicine, Brigham and Women's Hospital and Harvard Medical School, Boston, MA, USA

\section{Literatur}

1 Nathan C, Ding A. Nonresolving inflammation. Cell 2010; 140: 871 882

2 Serhan CN, Chiang N, Van Dyke TE. Resolving inflammation: dual antiinflammatory and pro-resolution lipid mediators. Nat Rev Immunol 2008; 8: 349 - 361

3 Serhan CN, Savill J. Resolution of inflammation: the beginning programs the end. Nat Immunol 2005; 6: 1191 - 1197

4 Karp CL, Flick LM, Park KW et al. Defective lipoxin-mediated anti-inflammatory activity in the cystic fibrosis airway. Nat Immunol 2004; 5: $388-392$

5 Celik GE, Erkekol FO, Misirligil Z, Melli M. Lipoxin A4 levels in asthma: relation with disease severity and aspirin sensitivity. Clin Exp Allergy 2007; 37: 1494-1501

6 Levy BD, Bonnans C, Silverman ES et al. Diminished lipoxin biosynthesis in severe asthma. Am J Respir Crit Care Med 2005; 172: 824-830

7 Tahan F, Saraymen R, Gumus $H$. The role of lipoxin A4 in exercise-induced bronchoconstriction in asthma. J Asthma 2008; 45: 161 - 164

8 Serhan CN, Hamberg M, Samuelsson B. Lipoxins: novel series of biologically active compounds formed from arachidonic acid in human leukocytes. Proc Natl Acad Sci U S A 1984; 81: 5335 - 5339

9 Serhan $C N$, Hamberg M, Samuelsson B. Trihydroxytetraenes: a novel series of compounds formed from arachidonic acid in human leukocytes. Biochem Biophys Res Commun 1984; 118: 943 - 949

10 Serhan $C N$. Resolution phase of inflammation: novel endogenous anti-inflammatory and proresolving lipid mediators and pathways. Annu Rev Immunol 2007; 25: 101 - 137

11 Fiore S, Serhan CN. Formation of lipoxins and leukotrienes during receptor-mediated interactions of human platelets and recombinant human granulocyte/macrophage colony-stimulating factor-primed neutrophils. J Exp Med 1990; 172: 1451 - 1457

12 Levy BD, Romano M, Chapman HA et al. Human alveolar macrophages have 15-lipoxygenase and generate 15(S)-hydroxy-5,8,11-cis-13trans-eicosatetraenoic acid and lipoxins. J Clin Invest 1993; 92: $1572-1579$

13 Claria J, Serhan CN. Aspirin triggers previously undescribed bioactive eicosanoids by human endothelial cell-leukocyte interactions. Proc Natl Acad Sci U S A 1995; 92: 9475 - 9479

14 Claria J, Lee MH, Serhan CN. Aspirin-triggered lipoxins (15-epi-LX) are generated by the human lung adenocarcinoma cell line (A549)-neutrophil interactions and are potent inhibitors of cell proliferation. Mol Med 1996; 2: $583-596$

15 Planaguma A, Pfeffer MA, Rubin G et al. Lovastatin decreases acute mucosal inflammation via 15-epi-lipoxin A4. Mucosal Immunol 2010; $3: 270-279$
16 Serhan CN, Fiore S, Brezinski DA, Lynch S. Lipoxin A4 metabolism by differentiated HL-60 cells and human monocytes: conversion to novel 15-oxo and dihydro products. Biochemistry 1993; 32: 63136319

17 Serhan CN, Maddox JF, Petasis NA et al. Design of lipoxin A4 stable analogs that block transmigration and adhesion of human neutrophils. Biochemistry 1995; 34: 14609-14615

18 Burr GO, Burr MM. Nutrition classics from The Journal of Biological Chemistry 82: $345-67,1929$. A new deficiency disease produced by the rigid exclusion of fat from the diet. Nutr Rev 1973; 31: $248-249$

19 Marchioli R, Barzi F, Bomba $E$ et al. Early protection against sudden death by $n-3$ polyunsaturated fatty acids after myocardial infarction: time-course analysis of the results of the Gruppo Italiano per lo Studio della Sopravvivenza nell'Infarto Miocardico (GISSI)-Prevenzione. Circulation 2002; 105: 1897-1903

20 Mickleborough TD, Lindley MR, Ionescu AA, Fly AD. Protective effect of fish oil supplementation on exercise-induced bronchoconstriction in asthma. Chest 2006; 129: 39-49

21 Freedman SD, Blanco PG, Zaman MM et al. Association of cystic fibrosis with abnormalities in fatty acid metabolism. N Engl J Med 2004; 350: 560 - 569

22 Serhan CN, Clish CB, Brannon J et al. Novel functional sets of lipid-derived mediators with antiinflammatory actions generated from omega-3 fatty acids via cyclooxygenase 2-nonsteroidal antiinflammatory drugs and transcellular processing. J Exp Med 2000; 192: 1197-1204

23 Serhan CN, Hong S, Gronert Ket al. Resolvins: a family of bioactive products of omega-3 fatty acid transformation circuits initiated by aspirin treatment that counter proinflammation signals. J Exp Med 2002; 196: 1025 - 1037

24 Wittamer V, Franssen JD, Vulcano M et al. Specific recruitment of antigen-presenting cells by chemerin, a novel processed ligand from human inflammatory fluids. J Exp Med 2003; 198: 977-985

25 Arita M, Bianchini F, Aliberti J et al. Stereochemical assignment, antiinflammatory properties, and receptor for the omega-3 lipid mediator resolvin E1. J Exp Med 2005; 201: 713- 722

26 Ohira T, Arita M, Omori K et al. Resolvin E1 receptor activation signals phosphorylation and phagocytosis. J Biol Chem 2010; 285: 3451 3461

27 Arita M, Ohira T, Sun YP et al. Resolvin E1 selectively interacts with leukotriene B4 receptor BLT1 and ChemR23 to regulate inflammation. J Immunol 2007; 178: 3912 - 3917

28 Hong S, Gronert K, Devchand PR et al. Novel docosatrienes and 17S-resolvins generated from docosahexaenoic acid in murine brain, human blood, and glial cells. Autacoids in anti-inflammation. J Biol Chem 2003; 278: 14677 - 14687

29 Serhan CN, Gotlinger K, Hong $S$ et al. Anti-inflammatory actions of neuroprotectin D1/protectin D1 and its natural stereoisomers: assignments of dihydroxy-containing docosatrienes. J Immunol 2006; 176: $1848-1859$

30 Duffield JS, Hong S, Vaidya VS et al. Resolvin D series and protectin D1 mitigate acute kidney injury. J Immunol 2006; 177: 5902 - 5911

31 Sun YP, Oh SF, Uddin J et al. Resolvin D1 and its aspirin-triggered 17R epimer. Stereochemical assignments, anti-inflammatory properties, and enzymatic inactivation. J Biol Chem 2007; 282: 9323 - 9334

32 Mattoscio D, Evangelista V, De Cristofaro R et al. Cystic fibrosis transmembrane conductance regulator (CFTR) expression in human platelets: impact on mediators and mechanisms of the inflammatory response. FASEB J 2010; 24: 3970 - 3980

33 Spite M, Norling LV, Summers L et al. Resolvin D2 is a potent regulator of leukocytes and controls microbial sepsis. Nature 2009; 461: 1287 1291

34 Krishnamoorthy S, Recchiuti A, Chiang $N$ et al. Resolvin D1 binds human phagocytes with evidence for proresolving receptors. Proc Natl Acad Sci USA 2010; 107: 1660-1665

35 Marcheselli VL, Hong S, Lukiw WJ et al. Novel docosanoids inhibit brain ischemia-reperfusion-mediated leukocyte infiltration and pro-inflammatory gene expression. J Biol Chem 2003; 278: 43807 - 43817

36 Lukiw WJ, Cui JG, Marcheselli VL et al. A role for docosahexaenoic acidderived neuroprotectin D1 in neural cell survival and Alzheimer disease. J Clin Invest 2005; 115: 2774-2783

37 Gonzalez-Periz A, Planaguma A, Gronert Ket al. Docosahexaenoic acid (DHA) blunts liver injury by conversion to protective lipid mediators: protectin D1 and 17S-hydroxy-DHA. FASEB J 2006; 20: 2537 - 2539 
38 Marcheselli VL, Mukherjee PK, Arita $M$ et al. Neuroprotectin D1/protectin D1 stereoselective and specific binding with human retinal pigment epithelial cells and neutrophils. Prostaglandins Leukot Essent Fatty Acids 2010; 82: 27-34

39 Serhan CN, Yang R, Martinod Ket al. Maresins: novel macrophage mediators with potent antiinflammatory and proresolving actions. J Exp Med 2009; 206: 15-23

40 Soehnlein 0 , Lindbom L. Phagocyte partnership during the onset and resolution of inflammation. Nat Rev Immunol 2010; 10: 427-439

41 Holgate ST. The epidemic of allergy and asthma. Nature 1999; 402: B2 - B4

42 Martinez Molina D, Wetterholm A, Kohl A et al. Structural basis for synthesis of inflammatory mediators by human leukotriene C4 synthase. Nature 2007; 448: 613-616

43 Busse WW, Lemanske RF Jr. Asthma. N Engl J Med 2001; 344: 350 362

44 Vachier I, Bonnans C, Chavis C et al. Severe asthma is associated with a loss of LX4, an endogenous anti-inflammatory compound. J Allergy Clin Immunol 2005; 115: 55-60

45 Sanak M, Levy BD, Clish CB et al. Aspirin-tolerant asthmatics generate more lipoxins than aspirin-intolerant asthmatics. Eur Respir J 2000; 16: $44-49$

46 Bonnans C, Vachier I, Chavis C et al. Lipoxins are potential endogenous antiinflammatory mediators in asthma. Am J Respir Crit Care Med 2002; 165: 1531 - 1535

47 Planaguma A, Kazani S, Marigowda G et al. Airway lipoxin A4 generation and lipoxin A4 receptor expression are decreased in severe asthma. Am J Respir Crit Care Med 2008; 178: 574- 582

48 Levy BD, Clish CB, Schmidt B et al. Lipid mediator class switching during acute inflammation: signals in resolution. Nat Immunol 2001; 2: $612-619$

49 Haworth O, Cernadas M, Yang Ret al. Resolvin E1 regulates interleukin 23 , interferon-gamma and lipoxin A4 to promote the resolution of allergic airway inflammation. Nat Immunol 2008; 9: 873-879

50 Lee TH, Crea AE, Gant V et al. Identification of lipoxin A4 and its relationship to the sulfidopeptide leukotrienes C4, D4, and E4 in the bronchoalveolar lavage fluids obtained from patients with selected pulmonary diseases. Am Rev Respir Dis 1990; 141: 1453-1458

51 Levy BD, De Sanctis GT, Devchand PR et al. Multi-pronged inhibition of airway hyper-responsiveness and inflammation by lipoxin $A(4)$. Nat Med 2002; 8: 1018-1023

52 Christie PE, Spur BW, Lee TH. The effects of lipoxin A4 on airway responses in asthmatic subjects. Am Rev Respir Dis 1992; 145: 1281 1284

53 Riordan JR, Rommens JM, Kerem B et al. Identification of the cystic fibrosis gene: cloning and characterization of complementary DNA. Science 1989; 245: 1066 - 1073

54 Anderson MP, Gregory RJ, Thompson S et al. Demonstration that CFTR is a chloride channel by alteration of its anion selectivity. Science 1991: 253: 202-205

55 Brennan S. Innate immune activation and cystic fibrosis. Paediatr Respir Rev 2008; 9: 271 - 279; quiz 279-280

56 Eickmeier O, Huebner M, Herrmann E et al. Sputum biomarker profiles in cystic fibrosis (CF) and chronic obstructive pulmonary disease (COPD) and association between pulmonary function. Cytokine 2010; 50: $152-157$

57 Khan TZ, Wagener JS, Bost T et al. Early pulmonary inflammation in infants with cystic fibrosis. Am J Respir Crit Care Med 1995; 151: 1075 1082

58 Bonfield TL, Panuska JR, Konstan MW et al. Inflammatory cytokines in cystic fibrosis lungs. Am J Respir Crit Care Med 1995; 152: 2111 2118

59 Konstan MW, Walenga RW, Hilliard KA, Hilliard JB. Leukotriene B4 markedly elevated in the epithelial lining fluid of patients with cystic fibrosis. Am Rev Respir Dis 1993; 148: 896-901

60 Hartl D, Latzin P, Hordijk Pet al. Cleavage of CXCR1 on neutrophils disables bacterial killing in cystic fibrosis lung disease. Nat Med 2007; 13: $1423-1430$

61 Kuo PT, Huang NN, Bassett DR. The fatty acid composition of the serum chylomicrons and adipose tissue of children with cystic fibrosis of the pancreas. J Pediatr 1962; 60: 394-403

62 Barnes PJ. Immunology of asthma and chronic obstructive pulmonary disease. Nat Rev Immunol 2008; 8: 183-192

63 Martins V, Valenca SS, Farias-Filho FA et al. ATLa, an aspirin-triggered lipoxin A4 synthetic analog, prevents the inflammatory and fibrotic effects of bleomycin-induced pulmonary fibrosis. J Immunol 2009; 182: $5374-5381$

64 Bernard GR, Artigas A, Brigham KL et al. The American-European Consensus Conference on ARDS. Definitions, mechanisms, relevant outcomes, and clinical trial coordination. Am J Respir Crit Care Med 1994; 149: $818-824$

65 Wheeler AP, Bernard GR. Acute lung injury and the acute respiratory distress syndrome: a clinical review. Lancet 2007; 369: 1553-1564

66 Ware $L B$, Matthay MA. The acute respiratory distress syndrome. N Engl J Med 2000; 342: 1334-1349

67 Mizgerd JP. Acute lower respiratory tract infection. N Engl J Med 2008; 358: $716-727$

68 Fukunaga K, Kohli P, Bonnans C et al. Cyclooxygenase 2 plays a pivotal role in the resolution of acute lung injury. J Immunol 2005; 174: $5033-5039$

69 Gilroy DW, Colville-Nash PR, Willis D et al. Inducible cyclooxygenase may have anti-inflammatory properties. Nat Med 1999; 5: 698-701

70 Schwartz J, Weiss ST. The relationship of dietary fish intake to level of pulmonary function in the first National Health and Nutrition Survey (NHANES I). Eur Respir J 1994; 7: 1821 - 1824

71 Schubert R, Kitz R, Beermann C et al. Effect of n-3 polyunsaturated fatty acids in asthma after low-dose allergen challenge. Int Arch Allergy Immunol 2009; 148: 321 - 329

72 Bettelli E, Oukka M, Kuchroo VK. T(H)-17 cells in the circle of immunity and autoimmunity. Nat Immunol 2007; 8: 345 - 350

73 Molet S, Hamid Q Davoine F et al. IL-17 is increased in asthmatic airways and induces human bronchial fibroblasts to produce cytokines. J Allergy Clin Immunol 2001; 108: 430-438

74 Park H, Li Z, Yang XO et al. A distinct lineage of CD4 T cells regulates tissue inflammation by producing interleukin 17 . Nat Immunol 2005 6: $1133-1141$

75 Cheung PF, Wong CK, Lam CW. Molecular mechanisms of cytokine and chemokine release from eosinophils activated by IL-17A, IL-17F, and IL-23: implication for Th17 lymphocytes-mediated allergic inflammation. J Immunol 2008; 180: 5625-5635

76 Gadek JE, DeMichele SJ, Karlstad MD et al. Effect of enteral feeding with eicosapentaenoic acid, gamma-linolenic acid, and antioxidants in patients with acute respiratory distress syndrome. Enteral Nutrition in ARDS Study Group. Crit Care Med 1999; 27: 1409-1420

77 Pacht ER, DeMichele SJ, Nelson JL et al. Enteral nutrition with eicosapentaenoic acid, gamma-linolenic acid, and antioxidants reduces alveolar inflammatory mediators and protein influx in patients with acute respiratory distress syndrome. Crit Care Med 2003; 31: 491 500

78 Pontes-Arruda A, Aragao AM, Albuquerque JD. Effects of enteral feeding with eicosapentaenoic acid, gamma-linolenic acid, and antioxidants in mechanically ventilated patients with severe sepsis and septic shock. Crit Care Med 2006; 34: 2325-2333

79 Singer $P$, Theilla $M$, Fisher $H$ et al. Benefit of an enteral diet enriched with eicosapentaenoic acid and gamma-linolenic acid in ventilated patients with acute lung injury. Crit Care Med 2006; 34: 1033-1038

80 Kang JX, Wang J, Wu L, Kang ZB. Transgenic mice: fat-1 mice convert n6 to $n-3$ fatty acids. Nature 2004; 427: 504

81 Mayer K, Kiessling A, Ott J et al. Acute lung injury is reduced in fat-1 mice endogenously synthesizing n-3 fatty acids. Am J Respir Crit Care Med 2009; 179: 474-483

82 Seki H, Fukunaga K, Arita $M$ et al. The anti-inflammatory and proresolving mediator resolvin E1 protects mice from bacterial pneumonia and acute lung injury. J Immunol 2010; 184: 836-843

83 Meduri GU, Kohler G, Headley S et al. Inflammatory cytokines in the BAL of patients with ARDS. Persistent elevation over time predicts poor outcome. Chest 1995; 108: $1303-1314$

84 Levy BD, Kohli P, Gotlinger Ket al. Protectin D1 is generated in asthma and dampens airway inflammation and hyperresponsiveness. J Immunol 2007; 178: 496-502

85 Colgan SP, Serhan CN, Parkos CA et al. Lipoxin A4 modulates transmigration of human neutrophils across intestinal epithelial monolayers. J Clin Invest 1993; 92: 75-82

86 Papayianni A, Serhan CN, Brady HR. Lipoxin A4 and B4 inhibit leukotriene-stimulated interactions of human neutrophils and endothelial cells. J Immunol 1996; 156: 2264 - 2272

87 Levy BD, Fokin VV, Clark JM et al. Polyisoprenyl phosphate (PIPP) signaling regulates phospholipase D activity: a 'stop' signaling switch for aspirin-triggered lipoxin A4. Faseb J 1999; 13: 903-911 
88 Soyombo O, Spur BW, Lee TH. Effects of lipoxin A4 on chemotaxis and degranulation of human eosinophils stimulated by platelet-activating factor and $\mathrm{N}$-formyl-L-methionyl-L-leucyl-L-phenylalanine. Allergy 1994; 49: 230-234

89 Bandeira-Melo C, Bozza PT, Diaz BL et al. Cutting edge: lipoxin (LX) A4 and aspirin-triggered 15-epi-LXA4 block allergen-induced eosinophil trafficking. J Immunol 2000; 164: 2267-2271

90 Ariel A, Chiang N, Arita M et al. Aspirin-triggered lipoxin A4 and B4 analogs block extracellular signal-regulated kinase-dependent TNFalpha secretion from human T cells. J Immunol 2003; 170: 62666272

91 Ramstedt U, Serhan CN, Nicolaou KC et al. Lipoxin A-induced inhibition of human natural killer cell cytotoxicity: studies on stereospecificity of inhibition and mode of action. J Immunol 1987; 138: $266-$ 270

92 Godson C, Mitchell S, Harvey K et al. Cutting edge: lipoxins rapidly stimulate nonphlogistic phagocytosis of apoptotic neutrophils by monocyte-derived macrophages. J Immunol 2000; 164: 1663-1667

93 Aliberti J, Hieny S, Reis e Sousa C et al. Lipoxin-mediated inhibition of IL-12 production by DCs: a mechanism for regulation of microbial immunity. Nat Immunol 2002; 3: 76-82

94 Gewirtz AT, McCormick B, Neish AS et al. Pathogen-induced chemokine secretion from model intestinal epithelium is inhibited by lipoxin A4 analogs. J Clin Invest 1998; 101: 1860 - 1869

95 Brezinski ME, Gimbrone MA Jr., Nicolaou KC, Serhan CN. Lipoxins stimulate prostacyclin generation by human endothelial cells. FEBS Lett 1989; 245: $167-172$

96 Bonnans C, Fukunaga K, Levy MA, Levy BD. Lipoxin A(4) regulates bronchial epithelial cell responses to acid injury. Am J Pathol 2006; 168: $1064-1072$

97 Campbell EL, Louis NA, Tomassetti SE et al. Resolvin E1 promotes mucosal surface clearance of neutrophils: a new paradigm for inflammatory resolution. Faseb J 2007; 21: $3162-3170$

98 Schwab JM, Chiang N, Arita M, Serhan CN. Resolvin E1 and protectin D1 activate inflammation-resolution programmes. Nature 2007; 447: $869-874$

99 Haworth O, Cernadas M, Yang R et al. Resolvin E1 regulates interleukin 23 , interferon-gamma and lipoxin $\mathrm{A}(4)$ to promote the resolution of allergic airway inflammation. Nat Immunol 2008; 9: 873-879

100 Ariel A, Fredman G, Sun YP et al. Apoptotic neutrophils and T cells sequester chemokines during immune response resolution through modulation of CCR5 expression. Nat Immunol 2006; 7: 1209-1216

101 Ariel A, Li PL, Wang Wet al. The docosatriene protectin D1 is produced by $\mathrm{TH} 2$ skewing and promotes human $\mathrm{T}$ cell apoptosis via lipid raft clustering. J Biol Chem 2005; 280: 43079-43086
102 Bannenberg GL, Chiang N, Ariel A et al. Molecular circuits of resolution: formation and actions of resolvins and protectins. J Immunol 2005; 174: 4345 - 4355

103 Mukherjee PK, Marcheselli VL, Serhan CN, Bazan NG. Neuroprotectin D1: a docosahexaenoic acid-derived docosatriene protects human retinal pigment epithelial cells from oxidative stress. Proc Natl Acad Sci U S A 2004; 101: 8491 - 8496

104 Fiorucci S, Wallace JL, Mencarelli A et al. A beta-oxidation-resistant lipoxin $\mathrm{A} 4$ analog treats hapten-induced colitis by attenuating inflammation and immune dysfunction. Proc Natl Acad Sci U S A 2004; 101: 15736 - 15741

105 Chiang N, Gronert K, Clish CB et al. Leukotriene B4 receptor transgenic mice reveal novel protective roles for lipoxins and aspirin-triggered lipoxins in reperfusion. J Clin Invest 1999; 104: 309-316

106 Fierro IM, Kutok JL, Serhan CN. Novel lipid mediator regulators of endothelial cell proliferation and migration: aspirin-triggered-15R-lipoxin A(4) and lipoxin A(4). J Pharmacol Exp Ther 2002; 300: 385 392

107 Svensson CI, Zattoni M, Serhan CN. Lipoxins and aspirin-triggered lipoxin inhibit inflammatory pain processing. J Exp Med 2007; 204: $245-252$

108 Serhan CN, Jain A, Marleau S et al. Reduced inflammation and tissue damage in transgenic rabbits overexpressing 15-lipoxygenase and endogenous anti-inflammatory lipid mediators. J Immunol 2003; 171: $6856-6865$

109 Gronert K, Maheshwari N, Khan N et al. A role for the mouse 12/15lipoxygenase pathway in promoting epithelial wound healing and host defense. J Biol Chem 2005; 280: 15267-15278

110 Hasturk H, Kantarci A, Ohira T et al. RvE1 protects from local inflammation and osteoclast-mediated bone destruction in periodontitis. Faseb J 2006; 20: 401 - 403

111 Hasturk H, Kantarci A, Goguet-Surmenian E et al. Resolvin E1 regulates inflammation at the cellular and tissue level and restores tissue homeostasis in vivo. J Immunol 2007; 179: 7021 - 7029

112 Connor KM, SanGiovanni JP, Lofqvist $C$ et al. Increased dietary intake of omega-3-polyunsaturated fatty acids reduces pathological retinal angiogenesis. Nat Med 2007; 13: $868-873$

113 Arita M, Yoshida M, Hong S et al. Resolvin E1, an endogenous lipid mediator derived from omega-3 eicosapentaenoic acid, protects against 2,4,6-trinitrobenzene sulfonic acid-induced colitis. Proc Natl Acad Sci U S A 2005; 102: 7671 - 7676

114 Weylandt KH, Kang JX, Wiedenmann B, Baumgart DC. Lipoxins and resolvins in inflammatory bowel disease. Inflamm Bowel Dis 2007; 13 : 797-799 